\title{
Autour du traité de Plutarque Vertus de femmes ( Gunaikôn Aretai)
}

Considerations around Plutarch's treatise The Virtues of Women (Gunaikon Aretai)

\section{Pauline SCHMITT PANTEL}

\section{(2) OpenEdition}

\section{Journals}

Édition électronique

URL : http://journals.openedition.org/clio/9369

DOI : 10.4000/clio.9369

ISSN : 1777-5299

Éditeur

Belin

Édition imprimée

Date de publication : 15 décembre 2009

Pagination : $39-60$

ISSN : 1252-7017

Référence électronique

Pauline SCHMITT PANTEL, "Autour du traité de Plutarque Vertus de femmes (Gunaikôn Aretai) 》, Clio. Histoire, femmes et sociétés [En ligne], 30 | 2009, mis en ligne le 15 décembre 2012, consulté le 20 avril 2019. URL : http://journals.openedition.org/clio/9369; DOI : 10.4000/clio.9369 


\title{
Autour du traité de Plutarque Vertus de femmes (Gunaikôn Aretai)
}

\author{
Pauline SCHMITT PANTEL
}

Timocléia, femme grecque de Thèbes, précipitant un capitaine thrace de l'armée d'Alexandre dans un puits, tête la première et jambes écartées, est le sujet d'un tableau d'Elisabetta Sirani, peint à Bologne en 16591. Cet acte héroïque de Timocléia est mis en scène par Plutarque à la fois dans la Vie d'Alexandre et dans les Vertus de femmes $^{2}$. Selon les deux récits qui diffèrent dans la forme mais non sur le fond, le soldat a violé Timocléia, une épouse vertueuse et la sœur d'un général de Thèbes, Théagénès, mort à Chéronée dans la bataille contre Alexandre, puis il a voulu s'emparer des richesses de la maison. Timocléia a prétendu que de l'or, des parures et de la vaisselle d'argent étaient dans un puits auquel elle l'a conduit. Poussé par sa cupidité, l'homme est descendu dans le puits où il a été enseveli et tué sous des pierres jetées par Timocléia et ses servantes. Après ce meurtre Timocléia, conduite devant Alexandre, lui déclare :

Théagène était mon frère, il est tombé à Chéronée, où il était stratège, en combattant contre vous pour la liberté de la Grèce, afin que nous n'ayons rien de tel à subir. Après les traitements indignes de notre race

1 Elisabetta Sirani (1638-1665): Timoclée précipite le capitaine d'Alexandre Magne dans le puits, 1659, Naples, Musée de Capodimonte, huile sur toile. Voir Hamon-Lehours 2009. Je remercie Emilie Hamon-Lehours de m'avoir permis de lire son article avant la publication.

2 Plutarque, Vie d'Alexandre, 12 et Moralia, 259 D-260 D. Timocléia est également citée par Plutarque dans les Préceptes Conjugaux: Moralia, 145 E dans une liste de femmes admirables et dignes de renom et dans le Non posse suaviter vivi secundum Epicurum (Moralia, 1093 C) où le récit de son acte est attribué à Aristoboule un auteur que connaissait Plutarque (Jacoby Frag. Gr. Hist. 139 f2.) 
que nous avons subis, nous ne fuyons plus la mort. Car il vaut mieux sans doute ne pas vivre une seconde nuit si tu ne dois pas les interdire $»^{3}$.

Alexandre fait libérer Timocléia et ordonne à ses officiers de faire cesser de tels outrages.

Entre le texte de Plutarque et le tableau qu'il a inspiré, quelque quatorze siècles se sont écoulés et Emilie Hamon-Lehours montre bien que, pour comprendre le sens de cette peinture en 1659, il faut tenir compte de la biographie singulière de cette jeune femme d'une famille patricienne, qui reçut une éducation la mettant en contact avec les auteurs antiques et qui choisit d'évoquer, dans sa peinture, des thèmes exaltant une certaine forme d'héroïsme féminin ${ }^{4}$.

Timocléia de Thèbes n'est pas présente dans la Cité des Dames de Christine de Pizan $^{5}$. Lorsqu'en 1404-1405 Christine compose son livre, elle a lu le De mulieribus claris de Boccace, dans sa version latine de 1361-1362, et peut-être dans sa traduction en français par un anonyme qui date de 1401: Des Clères et Nobles Femmes ${ }^{6}$. Elle ne connait sans doute pas les Vertus de femmes de Plutarque. Et il en est de même quand, un siècle plus tard, en 1504, Antoine Dufour écrit ses Vies des femmes célèbres à la demande d'Anne de Bretagne ${ }^{7}$. Parmi les quatre-vingt-onze «Vies» recensées par Antoine Dufour, on ne trouve en effet aucune des héroïnes retenues par Plutarque. Une des sources communes de Christine et d'Antoine Dufour est l'œuvre de Boccace. Or, les femmes dont Boccace trace l'histoire ne sont pas non plus celles qui apparaissent dans le traité de Plutarque ${ }^{8}$. Nombre

3 Plutarque, Moralia, 260 C (traduction Boulogne 2002).

4 Un autre tableau, inspiré de Plutarque, est étudié par Emilie Hamon-Lehours : Porcia se blessant à la cuisse, Houston, Stephen Warren Miles and Marylin Ross Miles Foundation, huile sur toile, 1664. L'auteur voit là une démarche personnelle d'Elisabetta Sirani qui veut « revaloriser le droit des femmes, leur autonomie et leur indépendance, ainsi que leur rôle politique dans la ville ».

5 Christine de Pizan, La cité des dames, texte traduit et présenté par Thérèse Moreau \& Eric Hicks, Paris, 1986.

6 Baroin \& Haffen 1993-1995.

7 Cassagnes-Brouquet 2007.

8 Boccace connaissait-il le traité de Plutarque? Les éditeurs du texte latin De mulieribus claris n'en sont pas sûrs et les éditeurs du texte en français n'en disent rien. 
d'entre elles appartiennent au monde grec et romain, mais les récits qui les concernent ont été transmis par d'autres auteurs antiques.

L'influence de Plutarque est, durant le Moyen Âge, quasiment inexistante en Occident ${ }^{9}$, mais tout change avec la restauration des études grecques au début du XVe siècle. Un premier recueil des Vies traduites en latin paraît en 1470, certains traités des Moralia sont traduits dès 1440 et, en 1485, paraît la traduction du De Virtutibus Mulierum par Alamanus Ranutinus (1429-1504) qui appartient à l'entourage de Laurent de Médicis. En mars 1509, les Aldes impriment à Venise la première édition grecque des Moralia, soit quatre-vingt-douze traités. Elle est faite à partir des manuscrits du cardinal Bessarion et due à la collaboration d'Erasme et de Demetrius Ducas. En 1572, Estienne donne une édition gréco-latine de Plutarque. Les traductions en langue moderne se sont succédé entretemps. Ce sont les Apophtegmes puis les Préceptes conjugaux qui semblent avoir été traduits les premiers en français; en témoigne le manuscrit attribué à Jean Lodé du Discours de Plutarque sur le mariage de Pollion et d'Eurydice écrit en 1499 à l'occasion des noces de Louis XII et Anne de Bretagne ${ }^{10}$.

Pour Les vertueux faits des Femmes, trois traductions sont antérieures à celle d'Amyot. D'abord, en 1538, celle de l'évêque humaniste de Bayonne Jean des Monstiers, dédiée à Marguerite de France et que Robert Aulotte qualifie de "traduction désinvolte». Puis, en 1546, celle du Champenois Denys Sauvage, seigneur du Parc, offerte en don à Anne Clavelle Galèse pour elle et ses amis. Enfin, celle de Claude de Tesserant, gentilhomme parisien, faite entre 1559 et 1572 et dédiée à Marguerite de France. On le remarque, ces trois traductions sont toutes dédiées à des dames de haut rang et de grande culture.

Amyot, après avoir traduit les Vies, se plonge à partir de 1565 dans les CEures Morales. Sa traduction parait en 1572 et elle est plusieurs fois rééditée avant sa mort en 1593. Elle connait une grande diffusion

9 Aulotte 1965. Sur la transmission des Moralia, voir également le Dictionnaire des philosophes antiques, art. « Plutarque », à paraître.

10 Voir sur tous ces aspects Aulotte 1965 qui donne les différentes versions manuscrites puis imprimées des Préceptes conjugaux et des Vertus de femmes. 
jusqu'en 162011. Robert Aulotte souligne son influence dans les domaines de la morale pratique et de la politesse mondaine - la pédagogie, la politique, la réflexion sur l'amour et l'amitié - et en littérature, des domaines qu'il associe à la morale pratique et à la politesse mondaine, et dont l'influence est également perceptible dans la littérature. «L'opuscule le plus souvent mis à contribution est celui des Vertueux faits de femmes: Montreux, Jean de Hays, Thomas Corneille y choisissent la dramatique histoire de Camma ; Hardy et Morel celle de Timoclée; Du Ryer retient l'aventure d'Arétaphile; et Rotrou celle de Chiomara dont il fait l'atroce et déjà tout romantique drame noir de Crisante $»^{12}$.

À l'époque moderne, le traité est utilisé aussi bien par les auteurs qui veulent défendre l'honneur du sexe féminin que par les « conservateurs » pour rappeler le rôle traditionnel des femmes. Il donne lieu à des lectures antithétiques ${ }^{13}$. Un exemple précis témoigne de l'usage qui en est fait. En Italie, à Piacenza, une traduction partielle et une édition des Vertus de Femmes paraît en $1794^{14}$. Ce livre constitue un cadeau de noces donné à l'occasion du mariage du noble Luigi dal Verme avec la comtesse Lucrezia Anguissola. Douze des récits de Plutarque seulement sont édités, ceux qui mettent en scène la piété et la fidélité conjugale. Comme le montre bien Valentina Paci, ce cadeau de noces est un miroir de son temps qui cadre parfaitement avec la cité de Piacenza alors très conservatrice.

On le voit, la question est autant de savoir si le traité de Plutarque a été connu et repris dans les écrits postérieurs, que d'étudier comment les actions de ses héroïnes ont été intégrées dans des modèles très différents, selon les contextes et les époques, pour finalement exprimer des valeurs qui, loin d'être éternelles, sont celles

11 Voir la réédition de la traduction d'Amyot des Moralia avec une notice sur les Vertus de femmes, éd. Champion, à paraittre. Je remercie Pascal Payen de m'avoir donné ces informations.

12 Aulotte 1965: 267-268. Pour un complément d'information sur l'époque classique et le XIXe siècle, voir les actes du colloque de Toulouse : Plutarque de l'âge classique au XIX siècle. Présences, interférences, dynamique, Toulouse Le Mirail, 13-15 mai 2009, à paraître.

13 Paci 2007-2008.

14 Paci 2008. 
d'un temps, d'une société, d'un genre littéraire précis ${ }^{15}$. C'est une enquête à mener qui suppose de bien connaître le contexte dans lequel écrit Plutarque et celui de l'œuvre qui a repris certains récits des Vertus de femmes. De façon plus limitée, je me contenterai ici de tirer un des fils qui permet de donner une cohérence et un sens au traité de Plutarque dans la culture grecque antique, ce qui permettra peutêtre ensuite à d'autres de comparer le but et la fonction de ce recueil particulier avec ceux qui mettent en scène les mêmes histoires, mais dans un contexte culturel différent.

\section{Le discours sur les femmes dans l'œuvre de Plutarque}

Plutarque est citoyen de la cité de Chéronée en Béotie en un temps où la Grèce est sous l'autorité de l'empire romain (46-120 après J.-C.). C'est un notable, prêtre d'Apollon au sanctuaire de Delphes et magistrat dans sa cité. Ses écrits, très nombreux, sont regroupés en deux ensembles: les Bioi ou Vies où Plutarque raconte de façon parallèle et en les comparant la vie d'un Grec et la vie d'un Romain, et les Moralia ou CEuvres Morales, qui rassemblent des traités de toute sorte. Les Vertus de femmes appartiennent à cet ensemble. Plutarque est considéré comme un philosophe moraliste qui tente de tirer des leçons du passé pour donner des conseils de conduite aux hommes de son temps ${ }^{16}$. Mais il adopte aussi la démarche de l'historien, travaillant sur des sources, tentant de comprendre des sociétés et des systèmes politiques fort éloignés des siens, voulant tirer de l'oubli les faits, institutions, gestes du passé grec et romain. Plutarque écrit au temps de l'empire romain et appartient à un moment intellectuel appelé la seconde sophistique qui redécouvre la Grèce classique et qui forge, à partir de cet héritage, une forme de pensée et de culture nouvelle.

15 Au début du XIXe siècle, par exemple, le livre de Catherine-Joseph-Ferdinand Girard de Propiac, Le Plutarque des jeunes demoiselles on Abrégé des vies des femmes illustres de tous les pays avec des leçons explicatives de leurs actions et de leurs ouvrages, Paris, 1806, est une adaptation féminine des œuvres de Plutarque.

16 Boulogne 1994 ; Frazier 1996 ; Sirinelli 2000 ; Plutarque 2001. 
Plutarque a traité dans son œuvre des figures féminines à plusieurs reprises ${ }^{17}$. En écrivant la vie des hommes illustres, il a dû parfois esquisser le portrait de leurs compagnes, ainsi celui d'Aspasie dans la vie de Périclès ${ }^{18}$. Il a également mis en scène des épisodes dans lesquels les femmes avaient un rôle majeur. Ainsi dans la Vie de Pélopidas, la mort d'Alexandre, tyran de Phères, est attribuée à l'action d'une femme, Thébè, fille de Jason et épouse du tyran ${ }^{19}$. Et dans la Vie de Cimon, l'histoire de Cléonice annonce la vengeance, à la fois divine et humaine, qui va s'abattre sur Pausanias ${ }^{20}$. En effet, le roi de Sparte envoie chercher cette jeune fille (parthenos) de Byzance, de naissance illustre, dans l'intention d'abuser d'elle. Cléonice entre dans la chambre de Pausanias dans l'obscurité, tombe et renverse une lampe. Le roi réveillé par le bruit et croyant qu'un ennemi l'attaque, tire son poignard et en frappe la jeune fille qui meurt. Thébè et Cléonice font partie d'une tribu d'héroïnes que Plutarque connaît bien et dont il a raconté l'histoire dans les Vertus de femmes ${ }^{21}$.

L'opinion des femmes grecques apparait aussi dans les Apophtegmes, leur conduite est au cœur de plusieurs Questions Grecques et Romaines et elles font l'objet de l'attention de Plutarque dans les Préceptes de mariage, la Consolation à sa femme, le Dialogue sur l'Amour, et les Histoires d'Amour entre autres ${ }^{22}$. Le traité appelé en grec Gunaikon Aretai, soit Mulierum Virtutes en latin, traduit en français, souvent mais pas toujours, par Vertus de femmes, leur est entièrement consacré. C'est un recueil de vingt-sept histoires mettant en scène des femmes, d'abord de façon collective (les Troyennes, les Phocidiennes...) puis de manière individuelle. Quelles femmes ? Plutarque l'indique dans le titre et dans le préambule de son ouvrage : il s'agit de femmes ayant fait preuve d'arété, un terme grec sur lequel il faut s'arrêter un instant.

17 Le Corsu 1981.

18 Schmitt Pantel 2007.

19 Plutarque, Pélopidas, 35, 5-12.

20 Plutarque, Cimon, 6, 6-7.

21 Plutarque, Moralia, 242 F- 263 C : Gunaikon Aretai. Stadter 1965. Boulogne 2002.

22 Tous ces traités sont édités dans la Collection des Universités de France (CUF), aux éditons des Belles Lettres, Paris. Les Histoires d'Amour relatent en fait cinq récits dont la teneur est très proche des histoires des Vertus de femmes. Pour une introduction d'ensemble aux Moralia, voir Flacelière \& Irigoin 1987. 
Il est souvent employé pour qualifier la conduite d'un homme : les Grecs parlent de l'arété d'Achille et avec lui de celle des héros homériques, comme de l'arété des Spartiates aux Thermopyles et avec eux de tout citoyen-soldat mourant pour sa patrie. Dans les écrits récents l'arété est souvent rendu par le terme de "valeur», une notion neutre et générale qui permet de glisser sous le terme des spécificités qui relèvent de l'époque, de la société, du régime politique. Or, dans le cas de ce traité de Plutarque, la traduction d'arété qui est choisie est le plus souvent celle de vertu. Une édition récente, celle de Jacques Boulogne dans la Collection des Universités de France, revient à l'expression grecque qu'il traduit ainsi: Conduites méritoires de femmes ${ }^{23}$. Ce titre à la tonalité médiévale préfère le terme de mérite à celui de vertu et, de ce fait, comme toute traduction, prend d'une certaine manière parti sur l'interprétation à donner à cette œuvre singulière. Il me semble que les termes «vertu » et « mérite» ont dans notre langue une connotation morale qui peut gauchir la notion grecque d'arété. Parler de la «valeur» des femmes permet de ne pas préjuger du contenu de cette arété, de se débarrasser pour un temps des poncifs qui s'attachent aux figures masculines et féminines et de lire ces récits avec un regard, sinon neuf, du moins de notre temps.

Plutarque a écrit des Vies des hommes illustres dans lesquelles il trace les contours de leur arété. Quel est son but en écrivant ce traité des Gunaikon aretai ? Il l'indique dans une préface de tonalité assez moderne, tout en dédiant son recueil à une amie du nom de Cléa :

Sur le mérite des femmes, Cléa, nous ne sommes pas de l'avis de Thucydide. En effet c'est celle dont on parle le moins hors de chez elle, en mal ou en bien, que pour sa part il déclare parfaite, car il estime qu’à l'instar de sa personne, le nom de la femme honnête doit être ainsi mis sous clé et ne pas sortir de chez elle. Gorgias nous parait plus nuancé, quand il enjoint de faire largement connaitre non le physique de la femme, mais sa réputation. Parfaite nous semble la coutume romaine, qui au nom de l'État rend aux femmes aussi, comme aux hommes, après leur décès, les éloges appropriées... J'ai rédigé pour toi le complément de nos propos sur la rigoureuse identité du mérite (arété) chez l'homme et chez la femme, complément où l'histoire sert de démonstration sans être arrangée pour le plaisir de l'oreille... Il n'est pas de meilleur moyen d'apprendre en quoi se ressemblent et se distinguent le mérite (arété)

23 Boulogne 2002. Les traductions citées ici sont celles de J. Boulogne. 
féminin et le mérite masculin que de mettre des vies en face de vies et des actions en face d'actions, comme des œuvres d'un grand art, tout en examinant si le goût de Sémiramis pour la grandeur (megalopragmosunê) a le même caractère et la même marque que celui de Sésostris, ou l'intelligence (sunesis) de Tanaquil que celle du roi Servius, ou la noblesse (phronema) de Porcia que celle de Brutus, ou celle de Timocléia que celle de Pélopidas, en prenant la notion de mérite dans son sens le plus général24.

Le programme de Plutarque est de comparer, dans le domaine de l'arété, femmes et hommes, programme étonnant dans le contexte de l'hellénisme et même dans le contexte du monde romain du $\mathrm{II}^{\mathrm{e}}$ siècle après J.-C. Pour le monde grec, le propos, attribué à Périclès en 430 avant J.-C. à la fin de l'oraison funèbre prononcée pour les premiers morts à la guerre du Péloponnèse, semble en effet avoir fait école :

Et s'il me faut rappeler à celles qui vivront désormais dans le veuvage quelles sont les vertus qu'on attend de leur sexe, j'exprimerais en une brève exhortation tout ce que j'ai à dire : on vous tiendra en haute estime si vous ne vous montrez pas inférieure à votre nature de femme et si vous vous conduisez de telle sorte que les hommes parlent de vous le moins possible, soit pour vous louer, soit pour vous critiquer ${ }^{25}$.

Plutarque prendrait-il dans son recueil le contre-pied de Périclès? C'est ce qu'il semble affirmer en disant: "nous ne sommes pas de l'avis de Thucydide ». Les écrits de Plutarque s'inscrivent, on le sait, dans le courant de la seconde sophistique. Selon ce courant de pensée philosophique et morale, chaque individu mérite reconnaissance et est destiné à un certain accomplissement de soi, les femmes comme les hommes ${ }^{26}$. Socrate et Antisthène, chacun à leur manière, ont reconnu la valeur des femmes comme celle des hommes, mais cette attitude intellectuelle n'est guère répandue dans la pensée des Grecs avant Plutarque ${ }^{27}$. La valorisation de la femme, surtout de la femme-épouse, se retrouve ainsi chez Plutarque dans les Préceptes de mariage, et dans le Dialogue sur l'Amour, comme dans le traité sur les Vertus de femmes. Les

24 Plutarque, Moralia, 242 e-f, 243.

25 Thucydide II, 45. Sur l'oraison funèbre : Loraux 1981 et sur le rôle des femmes dans l'histoire : Loraux 1989.

26 Wicker 1978.

27 Mac Inerney 2003. 
femmes que Plutarque choisit dans ce dernier traité s'illustrent par des comportements exemplaires de fidélité dans leurs relations privées ou familiales et également dans la communauté28. La femme de mérite n'a d'autre intérêt que le bien collectif et la conformité à un mode de cité où les hommes gouvernent et les femmes travaillent dans l'oikos à l'écart des affaires politiques. Les femmes des Gunaikon Aretai illustrent ainsi une forme de valeur qui s'exerce dans d'autres sphères que celle de la guerre. De façon plus générale, elles servent aussi le projet pédagogique de formation des individus qui est celui de Plutarque. Il remarque dans ce préambule que «les conduites méritoires doivent au caractère des différences d'un autre ordre, pour ainsi dire des colorations particulières... : différents sont le courage d'Achille et celui d'Ajax..., la grandeur d'âme de Cornelia n'est pas semblable à celle d'Olympias $»^{29}$. Même si les dissemblances individuelles ne créent pas pour autant plusieurs sortes de courage, de prudence ou de justice, il faut en tenir compte dans les récits.

Toutefois si Plutarque n'a pas écrit des Vies parallèles des femmes, je ne pense pas qu'il l'ait regretté et que ce traité soit une forme de compensation à l'absence de Vies des femmes illustres. Je pense plutôt que les femmes, même lorsqu'elles accèdent à la valeur (arété) chez Plutarque, ne peuvent prétendre à un discours semblable à celui qui est au cœur du genre du Bios consacré au destin des hommes. En effet, les Vies (Bioi) sont en grande partie construites autour de la place jouée par le politique dans la destinée de chaque personnage, or ce domaine reste interdit aux femmes, les Gunaikon Aretai le montrent clairement, même dans la construction imaginaire d'une forme d'héroïsme ${ }^{30}$.

\section{L'héroïsme dans les Vertus de femmes}

Le traité de Plutarque est un des rares textes grecs qui formule ce qui pourrait être une définition de l'héroïsme pour les femmes grecques. Il parle à propos d'Arétaphila de Cyrène: «d'un mérite

\footnotetext{
28 Sébillotte Cuchet 2008.

29 Plutarque, Moralia, 243 C-D.

30 Schmitt Pantel 2009a.
} 
(arété) dans sa conduite qui lui permet de rivaliser avec le Conseil des héroïnes $»^{31}$. Et au sujet de deux femmes galates, Stratoniké et Chiomara, de «deux femmes dignes de passer à la postérité »32. Enfin Timocléia de Thèbes qui a tué, nous l'avons vu, un soldat ayant abusé d'elle lors de la prise de la ville, suscite chez le conquérant Alexandre " un sentiment d'admiration $»^{33}$. Une conduite qui suscite l'admiration des hommes les plus célèbres pour son héroïsme, qui permet d'inscrire son nom dans la mémoire collective et qui fait entrer cette femme dans une sorte de Panthéon. Cette conduite, sanctionnée par la reconnaissance immédiate et durable des hommes et par celle des dieux, semble bien pouvoir être qualifiée d'héroïque.

Quels sont les gestes et les actes qui, aux yeux des Grecs, passent pour refléter l'arété des femmes dans ce traité et qui leur entrouvrent la porte de l'héroïsme ? Ce sont d'abord des leçons de courage. Les femmes rappellent aux hommes leur obligation d'andreia, de courage viril. Ainsi les femmes de Chios blâment leurs époux citoyens qui ont accepté, à la suite d'un traité, d'abandonner la ville avec pour tout bagage une chlamyde et un manteau, en se dépouillant de leurs armes.

Comme les hommes affirmaient s'y être engagés par un serment, elles leur enjoignirent de ne pas se défaire de leurs armes et de dire à l'ennemi que c'était la javeline et le bouclier qui étaient pour un homme de caractère sa couverture et sa tunique. Les colons de Chios obéirent et parlèrent sans détour aux Erythréens en leur montrant leurs armes... C'est donc parce que les femmes leur apprirent la hardiesse qu'ils furent sauvés de la sorte ${ }^{34}$.

Ce sont aussi des actes de courage. L'andreia des femmes de la cité d'Argos est connue par plusieurs auteurs ${ }^{35}$. Voici ce qu'en dit Plutarque :

Aucun des exploits réalisés collectivement par des femmes n'est plus illustre que le combat qu'elles menèrent contre Cléomène pour la

31 Plutarque, Moralia, $255 \mathrm{E}$ : boulè ton heroidon.

32 Plutarque, Moralia, 258 D. Le texte grec dit « dignes de mémoire » (mneme).

33 Plutarque, Moralia, 260 D. Le verbe employé : thaumaro signifie aussi l'étonnement d'Alexandre.

34 Plutarque, Moralia, 245 A.

35 Hérodote VI, 77 et 83. Pausanias II, 20, 8-9. Argos est une cité du nord-est du Péloponnèse. 
défense d'Argos à l'instigation de la poétesse Télésilla... Lorsque Cléomène, roi de Sparte, après avoir tué un grand nombre d'Argiens, marcha sur leur cité, un élan d'audace divine incita les femmes qui étaient dans la force de l'âge à s'efforcer de repousser l'ennemi pour défendre leur patrie. Sous la conduite de Télésilla, elles prirent les armes, et se tenant près des créneaux, elles couronnèrent le cercle des remparts, à la stupéfaction de l'ennemi. Le résultat fut qu'elles refoulèrent Cléomène en lui infligeant de grandes pertes... La cité ayant ainsi trouvé son salut, on enterra sur la Voie Argienne les femmes tombées au combat et il fut donné aux survivants, pour commémorer leur vaillance, d'ériger la statue d'Enyalios... Le jour anniversaire ils célèbrent jusqu'à présent la fête de l'Insolence (Hybristica), pendant laquelle ils font revêtir aux femmes des tuniques et des casaques d'hommes et aux hommes des robes et des voiles de femmes. Lorsque d'autre part ils cherchèrent à remédier au manque d'hommes, ce n'est pas aux esclaves, comme le rapporte Hérodote, qu'ils donnèrent les femmes en mariage, mais aux plus nobles des périèques, après leur avoir accordé le droit de cité. Or, même ceux-ci, elles paraissaient les mépriser et les dédaigner dans les relations conjugales, les tenant pour des inférieurs. Voilà pourquoi ils établirent une coutume qui prescrit aux femmes mariées l'obligation de porter une barbe pour dormir avec leur époux ${ }^{36}$.

Les femmes de la cité de Chios agissent de même à une autre occasion. Leur ville est assiégée par Philippe, fils de Démétrios, qui proclame l'union des esclaves et de leurs patronnes ${ }^{37}$. Alors, les femmes prises d'un dépit farouche vont sur les remparts, portent des pierres et des traits aux combattants, les exhortent, luttent personnellement à leurs côtés en envoyant une grêle de projectiles. Les ennemis sont ainsi repoussés. La violence des femmes est une marque de leur courage, sur lequel d'autres récits insistent. Les femmes de Mélos portent les armes sous leurs robes, armes dont se serviront leurs époux le moment venu pour tuer leurs hôtes, des Cariens, lors du banquet, et Plutarque commente: "Ce qu'il y a vraiment d'admirable, ce sont et le silence et la hardiesse des femmes et le fait que, sur un grand nombre, aucune, pas même involontairement, n'a, sous l'effet de la crainte, été lâche $»^{38}$.

36 Plutarque, Moralia, 245 C sq.

37 Plutarque, Moralia, 244 E sq.

38 Plutarque, Moralia, 247A. 
Les Tyrrhéniennes prennent la place de leurs époux dans les prisons de Sparte en échangeant leurs habits et «cela fait, les femmes restèrent en la place, s'étant préparées à toutes les atrocités ", allusion aux violences habituelles faites aux femmes ${ }^{39}$. Dans tous ces cas, et pour des raisons diverses, la survie de la communauté politique est en jeu. Les femmes font preuve d'andreia, de courage viril, voire de violence, mais c'est une violence acceptée par la cité ; toutefois les femmes qui y ont recours sont considérées comme des hommes, comme en témoigne bien la loi d'Argos.

Un autre trait de l'héroïsme féminin est dans ces récits l'acceptation, le renoncement, le consentement. Le plus bel exemple est celui des femmes de Phocide. Lors d'une guerre entre les Thessaliens et les Phocidiens, guerre d'anéantissement, les Phocidiens décident de rassembler tous leurs biens ainsi que leurs femmes et leurs enfants pour les brûler en un grand bûcher en cas de défaite. Mais comme un des Phocidiens suggérait de demander aux femmes leur avis, les femmes se réunirent en assemblée et prirent un décret adoptant la proposition faite par les hommes. «Et elles couronnèrent Daïphantos parce qu'il avait pris la décision la meilleure pour la Phocide $\gg^{40}$.

Les femmes peuvent aussi s'avérer bonnes conseillères en matière politique. Ainsi les Troyennes qui arrêtèrent l'errance de leur peuple parti de Troie en brûlant les vaisseaux, obligeant les hommes à s'établir en Italie ${ }^{41}$. Ou plus modestement la femme de Pythès qui oblige son époux à délaisser sa passion monomaniaque pour l'exploitation des mines d'or et lui suggère de diversifier les activités économiques dans son royaume. Le roi, retiré des affaires, c'est sa femme qui «administra le pouvoir avec sagesse $»^{42}$.

Enfin, les femmes font échec au cauchemar politique des cités grecques: la tyrannie ${ }^{43}$. Quatre histoires rapportées dans ce recueil ont pour sujet les femmes et le tyran. Elles sont d'importance inégale,

\footnotetext{
39 Plutarque, Moralia, 247C.

40 Plutarque, Moralia, 244D.

41 Plutarque, Moralia, 243E-244A.

42 Plutarque, Moralia, 263C.

43 Schmitt Pantel 2009c.
} 
mais elles forment un ensemble où les thèmes se répètent et se complètent. Ce sont l'histoire d'Eryxo, femme d'Arcésilas, et de Laarchos à Cyrène vers 570 avant J.C. ${ }^{44}$, l'histoire de Xénocrité et d'Aristodème à Cumes vers 504 avant J.C.45, de Megisto et d'Aristonimos, tyran à Elis pendant quelques mois vers 272 avant J.-C.46, l'histoire d' Arétaphila et de Nicocratès à Cyrène vers 96 avant J.C.47. Face au tyran, les femmes résistent par leur courage physique, leur habileté et leur parole politique. Elles suscitent la chute du tyran mais sont en revanche en retrait dans l'accomplissement de la vengeance et capables d'user de clémence et de refuser une punition indigne.

Un trait constant de ces récits est l'omniprésence de l'élément religieux qui apparaît de plusieurs manières : dans la fonction exercée par les femmes dont certaines sont prêtresses, dans les lieux et les moments où se déroule l'action valeureuse (sanctuaires, fêtes, rituels), et dans le geste méritoire même (libation empoisonnée, meurtre sur l'autel, rite de dérision efficace, suicide sur le bûcher sacrificiel ou funéraire ${ }^{48}$. L'arété des femmes leur vaut des honneurs qui, eux aussi, se situent dans la sphère cultuelle : funérailles, tombeaux, statues, rites après la mort. La familiarité des femmes dans le monde grec avec les rites et les dieux est totalement présente dans ces histoires. En effet, la religion tisse leur vie et il n'est pas surprenant qu'elle construise aussi leur arété.

\section{Le domaine politique : une frontière de l'héroisme}

Toutefois il est une frontière que même leur arété ne peut faire franchir aux femmes, celle du politique. Si l'on met en série leurs histoires, ce trait apparaît de façon constante : les femmes restent à la lisière du politique, leur destin s'arrête aux portes de la participation aux affaires de la cité. Soit elles meurent opportunément une fois leur exploit accompli, comme Polycrité de Naxos ou Lampsaké, soit elles

\footnotetext{
44 Plutarque, Moralia, 260D-261D.

45 Plutarque, Moralia, 261D-262D.

46 Plutarque, Moralia, 250F-253E

47 Plutarque, Moralia, 255 E -257 E.

48 Schmitt Pantel 2009 b.
} 
retrouvent leur condition de femme loin de la vie publique, après avoir décliné les honneurs (timai) et les présents (dorea), ainsi Xénocrité de Cumes et Arétaphila de Cyrène. Les citoyens de Cyrène sont libérés du tyran. «Le premier acte que leur inspira l'usage de la liberté fut de saluer Arétaphila avec des larmes de joie en se prosternant (prospiptein) comme aux pieds d'une statue (agalma theou) de déesse. Ils se succédaient les uns aux autres en un flot continu ${ }^{49}$. L'image est celle d'un rituel : celui de la procession vers la statue de la divinité. Puis le texte adopte le vocabulaire politique de l'éloge de l'évergète (bienfaiteur) : Arétaphila est honorée (timê) et louée (épainos) à satiété. Et les citoyens lui proposent le partage du pouvoir.

Ils voulurent qu'Arétaphila partageât avec l'élite la direction et l'administration des affaires (sunarchein, sundioikein ten politeian). Mais elle, après avoir pour ainsi dire joué un drame à péripéties et composé de plusieurs épisodes jusqu'au moment où lui fut attribuée la couronne, dès qu'elle vit la cité libre, se retira dans le gynécée et repoussant quelque ingérence que ce fût dans les affaires de l'État, elle passa tranquillement tout le reste de ses jours au milieu des métiers à tisser, en la compagnie de ses amis et de ses proches $^{50}$

Plutarque fait le choix pour Arétaphila d'une vie de tranquillité (hesuchia) contre la vie politique. Arétaphila devant son métier à tisser renoue avec une tradition qui remonte à Pénélope et façonne une image symbolique de la place dévolue aux femmes dans la cité. Le destin de Polycrité est une excellente illustration de cette incapacité des femmes à accéder aux honneurs politiques. Polycrité, une femme de Naxos qui permit aux siens de prendre par surprise la place forte détenue par les Milésiens, arrive aux portes de la ville :

Quant à elle-même, lorsqu'aux portes de la ville elle eut le spectacle de ses concitoyens qui venaient à sa rencontre, l'accueillant joyeusement avec des couronnes et lui témoignant leur admiration, elle ne supporta pas l'immensité de sa joie et tomba morte juste près de la porte. C'est là qu'elle est enterrée et l'endroit s'appelle le Tombeau-du-Mauvais-CEil, comme si c'était le sort qui avait regardé Polycrité d'un mauvais œil pour lui refuser par jalousie la jouissance des honneurs ${ }^{51}$.

\footnotetext{
49 Plutarque, Moralia, 257 C.

50 Plutarque, Moralia, 257 D-E.

51 Plutarque, Moralia, 254 E.
} 
Le commentaire de Jacques Boulogne ${ }^{52}$ porte sur la croyance des Grecs en l'existence de démons jaloux. Du coup l'expression «lui refuser par jalousie la jouissance des honneurs » est oubliée. Or, par cette mort, Polycrité est privée des honneurs attendus pour une évergète (bienfaitrice) et des honneurs possibles pour une héroïne. Du point de vue d'une histoire du genre, on peut remarquer que les «démons jaloux» du commentaire sont peut-être bien les citoyens eux-mêmes ou, de façon plus large, une pensée grecque masculine incapable de penser la place des femmes dans la vie politique autrement que sur le mode de la dérision. Plutarque (car l'auteur est derrière la remarque) transcrit là les limites de la pensée masculine de l'égalité entre les sexes. Cette mort est de plus présentée comme voulue par les divinités ou du moins par l'une d'entre elle : la Fortune n'est pas près de permettre le renversement des valeurs civiques. L'arété, la valeur, aussi grande soit-elle, ne peut effacer les frontières constitutives de la vie en cité, celles du genre et du politique.

Le cas de la femme de Pythès qui pourrait sembler être une exception, ne fait que confirmer cette règle. Après le départ de son mari dans le mausolée qu'il s'est construit et où il a l'intention de rester jusqu'à sa mort, "elle gouverna (archè) sagement et réforma ce qui rendait malheureux son peuple (métabolé kakon) » ${ }^{53}$. C'est le très rare exemple d'une femme qui par suite de son arété accède à une fonction politique, mais le récit se situe dans un royaume barbare, pas dans une cité grecque.

Ainsi les femmes sont « hérö̈ques » quand elles endossent l'andreia ou toute autre forme de valeur masculine. Mais cet héroïsme est de courte durée et tout rentre dans l'ordre quand le moment de crise extrême qui a failli voir la disparition de la cité prend fin. Les femmes reviennent à la maison et retrouvent un mode de vie approprié à leur sexe : le mariage, la procréation d'enfant, la part prise à l'économie domestique. Un rite vient parfois rappeler leur vaillance passée, mais il est aussi possible que leur acte héroïque ne puisse pas donner lieu à une forme de reconnaissance, comme le suggère la très curieuse fin de l'histoire de Polycrité. À la lecture des Vertus de femmes de

\footnotetext{
52 Boulogne $2002: 304$, n. 199.

53 Plutarque, Moralia, 262 D.
} 
Plutarque, il faut se résoudre à l'évidence: il n'est pas facile de devenir une héroïne dans le monde grec. Pourquoi ?

\section{Plutarque et au-delà}

Pour tenter de répondre à cette question, le traité de Plutarque doit être replacé dans la question plus générale de l'héroïsme au féminin dans le monde grec. Comme le suggère le thème de ce numéro de CLIO, il n'existe pas un invariant de l'hérö̈sme et donc une essence de la figure du héros et de l'héroïne à travers le temps et les espaces. Bien au contraire, les figures du héros et de l'héroïne sont des constructions sociales, contingentes, historiquement datées qui reflètent avant tout la société qui les a créées. Daniel Fabre, anthropologue et historien, a clairement mis en place la perspective selon laquelle on peut réfléchir au retour des héros dans le champ des curiosités historiques actuelles ${ }^{54}$. Il propose de s'interroger à la fois sur la figure hérö̈que, dans toutes ses composantes, et sur l'acte d'identification qui consacre cette figure comme paradigme aux yeux d'un groupe. On ne devient pas un héros ou une héroïne tout seul et de sa propre volonté. Cette promotion au rang de héros présuppose un accord tacite sur les valeurs qu'il incarne et repose sur une institutionnalisation des formes de mémoire qui vont produire son immortalité. Pour notre thème, l'héroïsme des femmes, ceci est important : la place moindre faite aux figures féminines de l'héroïsme peut être recherchée dans ce travail collectif de production; pour le dire autrement une société où les femmes ne sont pas les égales des hommes peut-elle penser l'héroïsme au féminin ?

L'inégalité entre les sexes règne dans le monde grec et ses traits essentiels sont bien connus. Ici je m'interrogerai seulement sur «le déficit d'images ", pour emprunter une expression à notre langage politique moderne, qui affecte les héroïnes grecques. En effet, les discours sur leur valeur sont de peu de poids face au discours dominant sur leur infériorité naturelle, et leurs actions sont beaucoup

54 Dans la conclusion d'un séminaire de l'école doctorale d'histoire de Paris 1 consacré au héros fondateur, Fabre 2002. Voir aussi Centlivres, Fabre \& Zonabend 1999. 
moins bien inscrites que celles des hommes dans la mémoire sociale, mémoire qui passe à cette époque souvent par le canal des rituels.

Les récits sur les héroïnes et sur les femmes qui semblent détenir quelque pouvoir ne sont qu'une toute petite partie de l'océan des discours que les Grecs ont produit sur les femmes, discours qui ont consisté avant tout à expliquer et justifier leur absence totale de droits. L'opposition entre un élément féminin qui serait du côté du monde sauvage et un élément masculin incarnant la culture, perdure pendant toute la civilisation antique et la dépréciation des femmes est à l'œuvre dans tous les types de discours avec de nombreuses nuances qu'il est impossible de rappeler ici. Dès la mise en place d'une réflexion sur la création des humains, le déséquilibre est manifeste puisque la première femme est «fabriquée », alors que les hommes existent déjà. Pandora, tel est son nom, est dès sa création toute artifice et tromperie, et avec elle toute la race maudite des femmes, ses descendantes, nous dit le poète archaïque Hésiode.

Dans les discours médicaux, en particulier les traités hippocratiques, la femme oscille entre le manque et l'excès, elle se définit par l'incomplétude et l'imperfection de chacun des éléments qui la composent ${ }^{55}$. C'est donc dans le cadre d'un discours, très construit et très systématique, de l'infériorité et de l'incapacité féminine qu'il convient de situer les textes qui mettent en scène, qui inventent des situations où les femmes semblent jouer un certain rôle. En dehors des récits qui sont colportés oralement et connus par un grand nombre, ou écrits et lus par une minorité, d'autres moyens existent pour donner à une figure de héros, légitimité et longévité. Le mode le plus important de mémorisation sociale dans ces cités de «face à face» comme les a appelées M.I. Finley est le rappel de l'identité et de l'histoire du héros lors de rituels perpétrés par la communauté civique toute entière ou par des subdivisions de celle-ci. La religion est, dans ces sociétés, indissociable de la création et de la promotion de toute figure héroïque. Or, la comparaison entre les cultes de héros et les cultes d'héroïnes est éclairante. En effet, pour les héroïnes, en raison du manque de documents en tout genre (textes et archéologie) les données que nous possédons dépassent rarement

$55 \quad$ King 1998. 
l'énoncé du nom et du lieu où l'héroïne est célébrée, le rituel est la plupart du temps non mentionné et l'on doit supposer que, dans leur dimension cultuelle, les héroïnes ne différaient guère des héros mais dans un genre mineur. Leur place est en effet largement subordonnée à celle des héros ${ }^{56}$. Elles apparaissent dans la position de compagnes et elles sont souvent anonymes. On les appelle seulement «l'épouse du héros ».

Pour devenir une héroïne, il faut que le contexte historique et social permette de faire de vous une figure exemplaire. Ou pour le dire autrement, tant que l'étoffe de l'héroïsme sera tissée par une cité andro-centrée, "un club d'hommes» comme l'écrit Pierre VidalNaquet, les femmes auront peu de chance d'être appelées " héroïnes » et leur arété ne sera reconnue que lorsqu'elle sera un exact décalque de celle des hommes. C'est ce que nous venons de voir avec les récits retenus par Plutarque dans son traité.

D’une manière générale, les rares récits dans lesquels les femmes semblent agir en faisant preuve d'héroïsme se placent dans un contexte particulier, celui de situations extrêmes: débuts des communautés, guerres extérieures menaçant d'anéantir totalement une cité, guerre civile aussi, fléau interdisant la reproduction des végétaux comme des animaux et des humains, où tout processus normal est bloqué. Ces récits ne semblent jamais mettre en place des sociétés où règnerait l'égalité entre les sexes, à l'exception très remarquable de la société des gardiens de la cité platonicienne de la République. Ils montrent tout au plus des sociétés qui, momentanément, connaissent le renversement en laissant aux femmes quelque pouvoir. Mais la fonction de ces récits est bien de montrer comment l'ordre a finalement été rétabli après un épisode aussi temporaire qu'en lui-même non créateur. Ainsi la vision fugitive d'un monde où les femmes auraient fait preuve d'hérö̈sme n'est qu'une des facettes du discours grec sur l'inégalité entre les sexes.

Et tout ceci est logique. Comment une société laissant aussi peu de droits et de pouvoirs aux femmes aurait-elle pu imaginer de

56 Ainsi en Attique comme le montre Kearns 1989. Une étude sur les héroïnes de l'Argolide en suivant Pausanias, aboutit aux mêmes conclusions. Voir de façon plus générale : Larson 1995, Pirenne-Delforge \& Suarez de la Torre 2000. 
manière durable un hérö̈sme des femmes? Quand les citoyens d'Argos permettaient à leurs femmes de porter la barbe le temps d'un rituel en souvenir d'un acte hérö̈que de leurs ancêtres, comme le rappelle Plutarque, ils ne pensaient pas pour autant qu'une société où les femmes aient des droits et des pouvoirs soit légitime. Et pour l'historienne aujourd'hui, il est intéressant de tenir compte à la fois de l'absence de droits et de pouvoirs des femmes dans la vie des cités et de ce discours masculin qui invente parfois des temps et des mondes où les femmes auraient eu accès à l'héroïsme. C'est ainsi que l'on peut tenter de comprendre quelle fut la place réelle et symbolique de l'inégalité entre les sexes dans l'Antiquité en se rappelant aussi que le principe constitutif des sociétés grecques a longtemps été l'exclusion, celle des femmes bien sûr, mais celle des autres aussi. Et, au delà du monde antique, comprendre comment l'inégalité entre les sexes construit historiquement une société, dans le domaine des représentations de l'hérö̈sme comme dans ses pratiques les plus banales et les plus ordinaires.

\section{Bibliographie}

AulotTe Robert, 1965, Amyot et Plutarque, La tradition des Moralia an XV Te siècle, Genève, Droz.

BARoIn Jeanne \& Josiane HAFFEn, 1993-1995, Boccace, «Des clères et nobles femmes », Besançon, Annales Littéraires de l'université de Besançon, 2 vol.

Blomqvist Katia, 1997, "From Olympias to Aretaphila. Women in Politics in Plutarch ", in Judith Mossman (ed.), Plutarch and his Intellectual World, Londres, Duckworth, p. 73-97.

Boulogne Jacques, 1994, Plutarque, un aristocrate grec sous l'occupation romaine, Lille, Presses universitaires de Lille.

-, 2002, Plutarque, Conduites méritoires des femmes, texte établi et traduit par J. Boulogne, Paris, Les Belles Lettres (CUF).

Cassagnes-Brouquet Sophie, 2007, Un manuscrit d'Anne de Bretagne. Les Vies des femmes célèbres d'Antoine Dufour, Rennes, Editions Ouest-France.

Centlivres Pierre, FABre Daniel \& Françoise ZonABend, 1999, La fabrique des héros, Paris, Éditions de la Maison des Sciences de l'Homme. 
FABre Daniel, 2002, «Le retour des héros », Hypothèses 2001, Paris, Publications de la Sorbonne, p. 225-228.

FlaCELIÈRE Robert \& Jean IRIGOIN, 1987, Plutarque, CEuvres Morales, tome I, introduction générale, Paris, Les Belles Lettres, p. I-CCCXXIV (CUF).

FRAZIER Françoise, 1996, Histoire et morale dans les Vies parallèles de Plutarque, Paris, Les Belles Lettres.

KEARns Emily, 1989, The Heroes of Attica, Londres, University of London, Institute of classical studies.

KING Helen, 1998, Hippocrates'Woman, Londres-New York, Routledge.

LARSON Jennifer, 1995, Greek Heroine Cults, Madison, University of Wisconsin Press.

Hamon-Lehours Emilie, 2009, «Plutarque, source d'inspiration de l'iconographie féminine. Elisabetta Sirani : entre art et littérature », colloque Plutarque de l'âge classique au XIXe siècle. Présences, interférences, dynamique, Toulouse Le Mirail 13-15 mai 2009, à paraître.

Le Corsu France, 1981, Plutarque et les femmes, Paris, Les Belles Lettres.

Loraux Nicole, 1981, L'invention d'Athènes, Paris, Mouton.

—, 1989, "Le naturel féminin dans l'histoire », in Les expériences de Tirésias, Paris, Gallimard, p. 273-300.

MAC InERnEY Jeremy, 2003, "Plutarch's Manly Women», in R.M. RoseN \& I. Sluiter (eds), Andreia, Studies in Manliness and Courage in Classical Antiquity, Leiden, Brill, p. 319-344.

PACI Valentina, 2007-2008, «Tradizione, novità e fortuna in età moderna del De mulierum virtutibus di Plutarco », Ploutarchos, n.s., p. 65-80.

—, 2008, «Le Virtù delle Donne di Plutarco in una traduzione piacentina di fine settecento », Bolletino Storico Piacentino, p. 81-101.

—, 2009, «Le rôle et l'influence de Plutarque dans la composition des Annales galantes de Grèce de Madame de Villedieu », colloque Plutarque de l'âge classique au XIXe siècle. Présences, interférences, dynamique, Toulouse Le Mirail 13-15 mai 2009, à paraître.

Pirenne-Delforge Vinciane \& Emilio Suarez de la Torre (dir.), 2000, Héros et béroïnes dans les mythes et les cultes grecs, Kernos, 10, Liège.

Plutarque, 2001, Vies Parallèles, édition publiée sous la direction de F. HarTog, Paris, Gallimard (Quarto).

SChmitt PANTEl Pauline, 2007, «Aspasie, la nouvelle Omphale. Genre, sentiment et politique au siècle de Périclès », in Pauline SCHMITT PANTEL \& François DE 
Autour du traité de Plutarque Vertus de femmes...

Polignac (textes réunis par), Athènes et le Politique, Paris, Albin Michel, p. 199221.

—, 2009a, Aithra et Pandora. Femmes, genre et cité en Grèce ancienne, Paris, L'Harmattan.

—, 2009b, «La religion et l'arété des femmes. À propos des Vertus de femmes de Plutarque ", in Lydie Bodiou \& Véronique MeHL (éd.), La religion des femmes en Grèce ancienne, Rennes, PUR, p. 145-159.

—, 2009c, «Les femmes vertueuses sont-elles des héroïnes ? Femmes et tyrans dans les Gunaikon Aretai de Plutarque », in P. Carlier \& C. Lerouge (dir.), Paysage et religion. Mélanges en l'honneur de Madeleine Jost (sous presse).

SÉBillotte-Cuchet Violaine, 2008, «Hérodote et Artémisia d'Halicarnasse, deux métis face à l'ordre des genres athéniens ", CLIO, Histoire, Femmes et Sociétés, 27, p. $15-33$.

Stadter Philipp, 1965, Plutarch's Methods. An analysis of the Mulierum Virtutes, Cambridge (Mass.), Harvard University Press.

WiCKER Kathleen, 1978, « Mulierum Virtutes (Moralia 242E-263C) », in Hans Dieter BETZ, Plutarch's Ethical Writings and Early Christian Litterature, Leiden, Brill, p. 106134. 